\title{
Abordagem diagnóstica de metástases hepáticas de câncer colorretal
}

\section{Diagnosis approach of liver metastases from colorectal cancer}

\author{
Paulo Afonso de Carvalho', William Abrão Saad²
}

\begin{abstract}
Carvalho PA, Saad WA. Abordagem diagnóstica e tratamento de metástases hepáticas de câncer colorretal. Rev Med (São Paulo). 2009; abr.-jun.;88(2):87-93.

RESUMO: A metástase hepática de câncer de colo e reto, uma doença cada vez mais comum e potencialmente agressiva, é discutida em âmbito internacional quanto à adequação da sua abordagem investigativa. Nesta revisão, este aspecto foi discutido tendo como parâmetros: custo, disponibilidade, sensibilidade, especificidade, uso de radiação e confiabilidade do método. A principal ideia é revisar, a partir de experiências adquiridas em diversos serviços de saúde, os principais métodos diagnósticos disponíveis para a detecção das lesões metastáticas no fígado originárias de câncer colorretal, discutindo em que situações eles devem ser utilizados e principalmente levando em conta a realidade do sistema médico hospitalar brasileiro.
\end{abstract}

DESCRITORES: Hepatectomia. Quimioterapia. Neoplasias hepáticas. Neoplasias de colo.

\section{INTRODUÇÃO}

$\mathrm{O}$ câncer de colo e reto é uma doença maligna muito comum em nosso meio, tendo prevalências maiores nas faixas etárias superiores da população, com quase $50 \%$ dos casos em pessoas com mais de 60 anos $^{24}$. No mundo ocidental, dentro dos óbitos ocasionados por câncer, ele é a segunda maior causa de morte em mulheres, enquanto, entre os homens, ele é a terceira maior causa de morte ${ }^{4}$.

Segundo dados do Instituto Nacional do Cân$\mathrm{cer}^{21}$, em 2008 o Brasil deve apresentar 12.490 casos novos entre os homens e 14500 casos novos entre as mulheres, valores que correspondem a um risco estimado de 13 novas ocorrências para cada 100.000 homens e 15 novas ocorrências para cada 100.000 mulheres, apontando uma tendência de crescimento da incidência frente os anos anteriores.

Quando comparados com os dados da Inglaterra (incidência de 58,1 a cada 100000 homens e 52,6 a cada 100.000 mulheres ${ }^{26,35}$, percebe-se a significativa diferença no aparecimento de novos casos nestes dois países, sendo normalmente mais freqüente em populações com nível sócio-econômico mais elevado.

\footnotetext{
1. Acadêmico do curso de medicina da Faculdade de Medicina da Universidade de São Paulo - FMUSP.

2. Professor Livre-Docente junto ao Departamento de Gastroenterologia da Faculdade de Medicina da Universidade de São Paulo - FMUSP, diretor do Serviço de Cirurgia do Fígado e Hipertensão Portal do Hospital das Clínicas da FMUSP.

Endereço para correpondência: Paulo Afonso de Carvalho. Rua Cardoso de Almeida, 703 - São Paulo, SP. CEP: 05013-000. E-mail: trackmed93@gmail.com
} 
A metástase do fígado é um evento frequente em pacientes com câncer colorretal, sendo a maior causa de morte nesta doença ${ }^{24}$. Isto ocorre porque este órgão é sítio preferencial de metástases hematogênicas provenientes de embolizações do sistema porta $^{8,15}$, através da veia mesentérica superior e da veia esplênica. Tais tumorações são passíveis de tratamento, sendo a ressecção cirúrgica a melhor opção, apresentando taxas de sobrevida pós-procedimento superiores a $40 \%{ }^{12,20,25,31}$. Porém, somente entre $20 \%$ a $25 \%$ dos pacientes com este problema têm indicação para a realização deste tratamento², por conta do comprometimento hepático avançado no momento do diagnóstico tardio.

Em algumas situações, o médico pode tratar o paciente com terapia de ablação, embolização através da veia porta, perfusão hepática isolada ou quimioterapia sistêmica ou intra-hepática por infusão arterial.

Dentre as pessoas que apresentam metástases hepáticas, aproximadamente $25 \%$ possuem o tipo sincrônico, ou seja, aquela em que as lesões hepáticas estão presentes no momento do diagnóstico ou no momento da ressecção do câncer colorretal, enquanto que de $40 \%$ a $50 \%$ desenvolvem o tipo metacrônico ${ }^{9}$, aquela que aparece após o tratamento cirúrgico do problema primário, normalmente dentro de um período de até 2 anos após o procedimento.

Infelizmente, as lesões metastáticas são responsáveis por até $90 \%$ das mortes nos primeiros três anos após a ressecção cirúrgica ${ }^{9}$. Tal agressividade, associada ao seu diagnóstico tardio, reforça a necessidade de se investir no desenvolvimento de metodologias mais efetivas na detecção precoce destas tumorações, bem como incentivar uma adequada investigação destas metástases no período pós ressecção cirúrgica, pois assim os cirurgiões fariam uma detecção precoce que acaba resultando em ressecções menores, aumentando a chance de cura destes pacientes.

Hoje, o aprimoramento de ferramentas de imagem, como ultrassonografia (US), laparoscopia, ressonância magnética $(\mathrm{RM})$, tomografia computadorizada (TC), tomografia com emissão de pósitrons com glicose ${ }^{18}$ FDG-Fluordeoxiglicose (FDG/ PET) e a integração de tomografia computadorizada com FDG/PET têm aumentado a capacidade dos médicos detectarem e caracterizarem focos de lesões hepáticas, resultando em melhorias da capacidade diagnóstica e de monitoramento deste problema ${ }^{7}$.

Além disto, abordagens promissoras, como TC com multidetectores (TCMD) e contrastes mais sensíveis e específicos para US, vêm sendo testados e aperfeiçoados ${ }^{32}$, oferecendo boas perspectivas para o futuro.
Entretanto, o número insuficiente de estudos, que determinam as melhores recomendações sobre quais e quando as abordagens diagnósticas devem ser utilizadas, acaba gerando desempenhos muito distintos entre os diversos hospitais ${ }^{2}$, fato que precisa ser discutido para se otimizar o número de diagnósticos desta doença utilizando as ferramentas que estão disponíveis no mercado.

Atualmente, alguns centros de pesquisa clínica no mundo, principalmente EUA e Holanda, têm apresentado preocupações neste sentido, e por isto já possuem um guideline $e^{3,5}$ que orienta os seus médicos sobre qual a melhor abordagem para a investigação de metástases hepáticas de câncer colorretal.

\section{Abordagem diagnóstica}

Uma vez diagnosticado o câncer colorretal em um paciente com alta concentração plasmática do antígeno carcinoembriogênico (CEA) (marcador tumoral ${ }^{16,17}$ altamente expresso em tumores do trato gastrointestinal), é fundamental que o médico inicie uma investigação para eventuais metástases hepáticas.

Trabalhos como o de Hostetter et al. ${ }^{19}$ evidenciaram que, após transplantes de fígado em ratos, o número de neoplasias secundárias no órgão subia de $2 \%$ para $48 \%$ nos animais que recebiam injeções com CEA, demonstrando a importância deste antígeno no seguimento da disseminação tumoral, mesmo não havendo evidências diretas que suportem esta afirmação.

Eventualmente, o prosseguimento da investigação pode revelar líquido ascítico com elevado teor protéico e grande concentração de desidrogenase lática. A dosagem sérica de albumina pode estar normal ou discretamente diminuída, e os níveis séricos de fosfatase alcalina, bilirrubina total e desidrogenase lática podem estar aumentados ${ }^{33}$.

A seguir, métodos de imagem devem ser requeridos para o seguimento da pesquisa. Hoje, a literatura ainda não propõe uma abordagem única e consensual que determine qual método de imagem é o padrão ouro para a detecção de lesões secundárias no fígado, tampouco qual seqüência de técnicas e em que situações elas devem ser empregadas com o mesmo propósito.

A ausência desta linha de conduta, previamente determinada (guideline) leva o médico a consumir muito tempo para diagnosticar a metástase hepática ${ }^{32}$ e a variar muito a sua abordagem investigativa ${ }^{2}$, usando múltiplos métodos diagnósticos ${ }^{32}$, fato extremamente oneroso para o sistema público de saúde, uma vez que acaba-se utilizando técnicas inadequadas para a detecção de um problema que, eventualmente, 
poderia ter sido detectado em um menor número de exames. Além disto, o profissional de saúde acaba adotando estratégias investigativas baseando-se em preferências e experiências profissionais e institucionais equivocadas, o que muitas vezes resulta em escolhas variáveis e inconsistentes ${ }^{2}$.

Finalmente, o médico acaba não tendo um parâmetro palpável para avaliar se sua conduta foi a ideal ou não para o caso em análise, o que é extremamente perigoso, pois idéias errôneas de raciocínio diagnóstico podem acabar sendo difundidas como abordagens corretas.

Por conta disto, o conhecimento dos principais pontos positivos e negativos de cada método frequentemente utilizado na detecção de metástases hepáticas e a organização de estudos que sumarizem o que já está proposto na literatura são fundamentais para entendimento e desenvolvimento de uma seqüência interessante e factível de métodos diagnósticos para cada realidade.

A seguir serão apresentadas as principais abordagens utilizadas na pesquisa de metástase hepática de câncer colorretal.

\section{Ultrassonografia}

A ultrassonografia transabdominal é o método mais utilizado no rastreamento primário de neoplasias hepáticas oriundas de câncer colorretal. Tal fato ocorre pelas seguintes facilidades: imagem em tempo real, baixo custo, abordagem não invasiva, ausência de radiação, imagens seccionais obtidas de qualquer plano e difusão da técnica pelos centros médicos. No entanto, problemas como necessidade de considerável experiência do ultrassonografista, resultados equivocados em pacientes com esteatose e sensibilidade e especificidade inferiores aos dos outros métodos ${ }^{32}$ levantam discussões sobre os seus benefícios na triagem inicial destas disseminações pelo fígado, uma vez que, em situações de lesão indeterminada, acaba sendo necessária a utilização de métodos mais precisos para esta caracterização ${ }^{11}$.

Nos EUA, onde a disponibilidade de RM e TC é grande e o número de ultrassonografistas qualificados é proporcionalmente baixo para seu território, o papel da US como método de imagem diagnóstico de metástases hepáticas é cada vez menor ${ }^{5}$, ficando relegada a pequenos centros que não possuem RM e TC, servindo como avaliação inicial antes de encaminhar este paciente para um hospital com infraestrutura melhor.

Porém, o desenvolvimento de contrastes, que aumentam a sensibilidade e especificidade de $78 \%$ para $100 \%$ e de $23 \%$ para $92 \%$ respectivamente; o uso da ultrassonografia intra-operatória (USIO), mé- todo mais acurado para a detecção de metástases hepáticas ${ }^{39,30}$ e a utilização de US associada com laparoscopia têm permitido melhor avaliação das lesões metastáticas ${ }^{38}$, viabilizando a detecção de um maior número de pequenas lesões malignas ou benignas, com o inconveniente de estas duas últimas abordagens serem invasivas.

\section{Tomografia computadorizada}

A TC é um método que vem se popularizando no meio médico, sendo particularmente interessante para detectar doenças metastáticas, pois consegue avaliar o fígado e eventuais tumorações extrahepáticas em um mesmo exame, com especificidade $90 \%$ e sensibilidade de $87 \%{ }^{40}$.

Nos EUA, a TC helicoidal é o exame predileto para acompanhamento de metástases no fígado após o tratamento da neoplasia primária colorretal. Como a maioria dessas lesões são hipovasculares, quando comparadas com o parênquima hepático, as imagens são hipoatenuantes quando geradas durante a fase portal ${ }^{5}$. Portanto, na maioria dos casos, esta fase é adequada para demonstrar este tipo de doença ${ }^{6}$, o que não permite que a fase arterial seja negligenciada no exame, pois ela pode detectar tumorações hipervasculares e também aumentar a conspicuidade das lesões hipovasculares em um pequeno número de casos $^{13}$.

Com a evolução das TC com multidetectores, o fígado pode ser copiado em cortes milimétricos em menos de 3 segundos, podendo ser reconstruído em imagens com boa resolução espacial, fato que otimiza a detecção da enfermidade.

Como pontos negativos da tomografia, temos o fato dela usar radiação para obtenção das imagens, ser um exame invasivo (com uso de contraste), relativamente cara e não estar disponível em todos os centros médicos, fato particularmente importante no Brasil.

\section{Ressonância magnética}

A RM é um método de imagem que tem papel importante na detecção e caracterização de lesões hepáticas ${ }^{29}$

A partir da introdução da spin-echo T1 e T2, rápidas seqüências de imagens passaram a ser obtidas, permitindo que o fígado fosse avaliado sem a presença de artefatos em um curto intervalo de tempo em que o paciente fica em apnéia forçada.

Em uma RM, as metástases hepáticas são vistas com hipossinal em T1 e hiperssinal em T2. Entretanto, muitas vezes estas lesões se apresentam com intensos sinais heterogêneos, com margens 
dismorfas e indistintas, podendo também aparecer como uma massa central irregular com hiperssinal, rodeada por um anel com hipossinal ou mesmo cercada por um anel com hiperssinal ${ }^{5}$.

Diversos estudos têm comparado a acurácia das diversas vertentes da RM frente a outros métodos. Um grande trabalho, do Radiology Diagnostic Oncology Group comparou TC e RM na detecção de metástases hepáticas oriundas de câncer colorretal ${ }^{41}$. Nele, a TC apresentou uma sensibilidade maior e especificidade semelhante à RM.

Já com relação ao uso ou não do gadolínio, existe uma discussão intensa sobre seu valor nesta abordagem diagnóstica, uma vez que existem trabalhos divergentes sobre o assunto.

Segundo Soyer ${ }^{34}$, o uso de gadolínio proporciona a obtenção de imagens de lesões secundárias no fígado de câncer colorretal com qualidade superior, ao passo que Hamm ${ }^{18}$ obteve resultados totalmente distintos, obtendo melhores imagens do mesmo problema, sem o uso deste contraste.

Por isto, a discussão sobre o uso ou não de gadolínio para a detecção de metástases hepáticas de câncer colorretal ainda não está terminada, precisando de mais evidências para concluir se ele tem valor diagnóstico neste caso, embora ninguém discuta sua importância na diferenciação deste problema com os hemangiomas.

Entretanto, o uso de gadolínio quelado é particularmente importante na distinção entre uma lesão metastática e um hemangioma, pois acentua os sinais obtidos em T1 após sua fase de equilíbrio de distribuição. Desta forma, o hemangioma, que permanece com uma poça deste produto, apresenta uma imagem com hiperssinal em T1, ao passo que as metástases aparecem como vem descrito anteriormente ${ }^{1}$.

As principais desvantagens da RM no diagnóstico destas neoplasias secundárias no fígado são: valor do exame (mais caro do que as outras metodologias citadas), abordagem eventualmente invasiva pelo uso de contrastes, sensibilidade inferior a da TC e disponibilidade restrita pelos centros médicos.

Tomografia com emissão de pósitrons com glicose ${ }^{18}$ FDG-Fluordeoxiglicose

A FDG/PET é um exame que distingue neoplasias através da detecção de diferenças na atividade metabólica celular. Nestas células, existe uma taxa de consumo de glicose maior do que em células normais ${ }^{22}$, fato que pode ser percebido com a ajuda de moléculas de glicose fluordeoxiglicose, marcada com isótopo radioativo de fluorina, que são captadas pelo tumor, mas não são consumidas por ele, fazendo com que este composto se acumule mais nestas células.

$\mathrm{O}$ isótopo sofre um decaimento radioativo emitindo pósitrons, que, por sua vez, reagem com elétrons adjacentes, produzindo 2 raios gamas de baixa energia que são quantificados por detectores para finalmente gerar uma imagem ${ }^{40}$. Por conta disto, a FDG/PET é conhecida como exame funcional, apresentando baixa qualidade anatômica.

Para solucionar este problema, imagens de TC do paciente são obtidas e colocadas sobre as imagens da FDG/PET, permitindo um melhor detalhamento anatômico, fato imprescindível para o sucesso desta abordagem médica. Tal associação de técnicas é conhecida como PET-CT ${ }^{40}$.

$\mathrm{Na}$ avaliação das metástases hepáticas, o FDG/PET apresenta uma sensibilidade de 94\% e uma especificidade de $98 \%{ }^{40}$, números sugestivos da acurácia desta metodologia.

Segundo Vitola ${ }^{37}$, em um teste duplo-cego o FDG/PET apresentou sensibilidade similar à TC na detecção de metástases hepáticas. Além disto, existem trabalhos como o de Delbeke ${ }^{10}$ que demonstram que o FDG/PET é mais sensível para a detecção de lesões extra-hepáticas do que a TC.

A partir destes dados, parece que FDG/ PET pode gerar informações mais relevantes no planejamento da terapêutica deste tipo de tumor do que as técnicas convencionais (US, RM e TC), além de ter papel de destaque no seguimento de tratamentos paliativos como crioterapia e ablação por radiofrequência.

No entanto, existem trabalhos ${ }^{3}$ que questionam a utilização do FDG/PET como método diagnóstico de lesões metastáticas no fígado de câncer colorretal. Segundo estes estudos, os dados na literatura ainda são preliminares e precisam de mais ensaios clínicos que confirmem seu potencial para a referida finalidade.

Outros inconvenientes são: elevado preço; baixa disponibilidade de equipamentos; uso de radiação; resolução espacial limitada, reportando-se falsos negativos em pequenas lesões ${ }^{14}$; o fato de ser invasivo; problemas para identificação de neoplasias com baixa atividade metabólica ${ }^{28}$ e dificuldade na logística de transporte da glicose marcada do local de produção até o hospital, uma vez que a pequena meia-vida do radioisótopo obriga que o centro produtor da molécula radioativa seja relativamente próximo do local de sua utilização.

\section{DISCUSSÃO}

Em tempos de verba limitada para os cuidados 
com a saúde, há um considerável debate sobre qual modalidade de exame de imagem não invasivo apresenta a melhor relação custo benefício para se avaliar metástases hepáticas oriundas de câncer colorretal, oferecendo capacidade de detecção e caracterização dos focos de lesão neste órgão ${ }^{32}$.

Países como EUA e Holanda, que já possuem um guideline sobre a investigação deste tipo de doença ${ }^{3,5}$, exibem posições distintas sobre esta questão.

Bipat et al. ${ }^{3}$, organizadores do trabalho de desenvolvimento do guideline holandês, propuseram que o primeiro passo na investigação desta problemática é a determinação do nível plasmático de CEA e o pedido de uma US transabdominal. Caso os resultados destes exames não apresentem anormalidades, este paciente será apenas acompanhado. Porém, caso a US mostre lesões suspeitas, o passo seguinte é o pedido de uma TC helicoidal ou RM do fígado com contraste, para assim melhor evidenciar as lesões e também avaliar as condições de ressecabilidade deste órgão.

Sendo diagnosticadas metástases potencialmente ressecáveis, o médico deve utilizar no intraoperatório a laparotomia associada à US para finalmente definir a viabilidade de execução da cirurgia.

Ainda segundo esta avaliação de Bipat, os dados sobre o potencial do FDG/PET na determinação de metástases hepáticas e parâmetros de ressecabilidade são limitados e prematuros, precisando de mais trabalhos para confirmá-los e assim possibilitar sua utilização como uma abordagem rotineira na prática médica.

Já nos EUA, o American College of Radiology recomenda, após a determinação de elevados níveis plasmáticos de CEA, a utilização de TC helicoidal do abdome como melhor passo para iniciar a investigação deste tipo de metástase hepática e, na persistência de dúvida, a orientação é que os médicos utilizem a RM em avaliação complementar ${ }^{5}$.

A ausência da realização da US nesta fase inicial de procura das lesões metastáticas é uma diferença importante nas condutas diagnósticas adotadas nos centros médicos destas duas populações, evidenciando o papel secundário que esta metodologia tem nos EUA ${ }^{5}$, principalmente por conta da grande difusão de tomógrafos neste país.

No entanto, o desenvolvimento de contrastes ultrassonográficos, que aumentam sensibilidade e especificidade para a detecção destas neoplasias hepáticas secundária ao câncer colorretal ${ }^{32}$, parece ser uma ferramenta promissora que possivelmente irá resgatar a importância da US transabdominal nos EUA, podendo futuramente substituir a TC helicoidal como primeira opção de escolha para investigação desse problema.

Ainda dentro dos EUA, o Conference on the Management of Hepatic Colorectal Metastases, organizado pelo American Hepato-Pancreato-Biliary Association (AHPBA), juntamente com a Society for Surgery of the Alimentary Tract e o Gastrointestinal Symposium Steering Committee ${ }^{36}$, recomendou o uso de rotina do FDG/PET antes de cirurgias de ressecções de metástases hepática. Segundo esta reunião, inúmeros trabalhos demonstram que o FDG/PET (com TC) é o exame mais sensível para a detecção de neoplasias secundárias no fígado ${ }^{19,12}$, fato que viabilizaria sua utilização corriqueira nos hospitais. Porém, o elevado custo (cerca de 3 mil dólares por paciente ${ }^{27}$ ) associado com as boas taxas de detecção de outros métodos, faz com que o FDG/PET ainda não seja um consenso dentro deste país.

Embora distintos, o principal valor destes dois guidelines é buscar homogeneizar e orientar as condutas investigativas para metástases hepáticas oriundas de câncer colorretal em suas populações, evitando variabilidade e procurando aumentar a eficiência do serviço, conseguindo realizar diagnóstico precoce deste tipo de problema.

Fazendo um paralelo com a realidade do Sistema Único de Saúde do Brasil, é plausível aceitar que o trabalho holandês apresente uma seqüência de metodologias diagnósticas que melhor atende às limitações de infra-estrutura, financeiras e técnicas vivenciadas nos diferentes centros médicos brasileiros, propondo uma ordenação seriada de métodos investigativos de acordo com uma ordem crescente de custo e acurácia, que procura não onerar o serviço público e ainda manter a excelência da capacidade investigativa deste tipo de neoplasia.

Em centros médicos que não ofereçam condições satisfatórias de diagnosticar este tipo de problema, é razoável que a equipe médica responsável não tente nenhuma abordagem cirúrgica investigativa. Nestas situações, o ideal é que se encaminhe este paciente para um hospital com infra estrutura adequada, para então iniciar a pesquisa do problema o mais rápido possível.

Portanto, embora o aprimoramento do tratamento dos pacientes com este tipo de metástase seja imperativo, este não depende exclusivamente da melhoria das técnicas curativas destas lesões. Ele também passa pelo desenvolvimento de métodos de imagem com maior sensibilidade e especificidade para realizar a detecção tumoral.

Finalmente, deve ser incentivada a proposição e adoção de um guideline de investigação que contenha os principais aspectos da realidade 
brasileira; que devem ser avaliados pelos médicos em suspeita desta enfermidade, balizando as condutas médicas, de forma que os resultados finais de tratamento sejam mais consistentes, reprodutíveis e homogêneos.

\section{CONCLUSÕES}

A partir dos dados levantados por esta revisão, o tratamento dos pacientes com metástases hepáticas de câncer colorretal no Brasil deve partir da utilização da US transabdominal como primeiro método de imagem para a detecção desta doença. No passo seguinte da investigação, a TC helicoidal ou a RM com contraste são as opções mais adequadas para o seguimento de avaliação destas lesões no fígado.

Quanto à utilização do FDG/PET como ferramenta de rotina no diagnóstico deste tipo de problema, o presente grupo concorda com Bipat et al. $^{3}$, optando por aguardar a criação de condições técnicas, financeiras e de infra-estrututa para que futuramente esta abordagem tenha sua realização viável na rotina da prática médica brasileira.

Finalmente, é importante que se incentive a proposição de um protocolo que estabeleça as principais condutas a serem adotadas pela equipe médica em casos de suspeita desta patologia, objetivando tratamentos mais adequados, com conseqüentes melhorias nas taxas de sobrevida dos pacientes.

Carvalho PA, Saad WA. Diagnosis approach of liver metastases from colorectal cancer. Rev Med (São Paulo). 2009 abr.-jun.;88(2):87-93.

\begin{abstract}
The liver metastasis of cancer of the colon and rectum, a disease increasingly common and potentially aggressive, is discussed at the international level about the adequacy of its investigative approach. In this review, this parameter was analysed with the cost, availability, sensitivity, specificity, use of radiation and reliability of the method. The main idea is to review, from experiences in various health services, the main diagnostic methods available for the detection of metastatic lesions in the liver unique for colorectal cancer, discussing situations in which they should be used and especially taking into to the reality of the Brazilian hospital medical system.
\end{abstract}

KEY WORDS: Hepatectomy. Drug therapy. Liver neoplasms. Colonic neoplasms.

\section{REFERÊNCIAS}

1. Bennett GL, Petersein A, Mayo-Smith WW, Hahn PF, Schima W, Saini S. Addiction of gadolinium chelates to heavily T2-weighted MR imaging: limited role in differentiating hepatic hemangiomas from metastases. AJR Am J Roentgenol. 2000;174:477-8.

2. Bipat S, Leeuwen MS van, ljzermans JNM, Bossuyt PMN, Greve JW, Stoker J. Imaging and treatment of patients with colorectal liver metastases in the Netherlands: a survey. Neth J Med. 2006;64:147-51.

3. Bipat S, Leeuwen MS van, ljzermans JNM, Comans EFI, Planting ASTh, Bossuyt PMM, et al. Evidence-based guideline on management of colorectal liver metastases in the Netherlands. Neth J Med. 2007;65(1):5-14.

4 Braet F, Nagatsuma K, Saito M, Soon M, Wisse E, Matsuura AT. The hepatic sinusoidal endothelial lining and colorectal liver metastases. World J Gastroenterol. 2007;13(6):821-5.

5 Bree RL, Greene FL, Ralls PW, Balfe DM, DiSantis DJ, Glick SN, et al. Suspected liver metastases. American College of Radiology. ACR appropriateness criteria. Radiology. 2000;215(Suppl):213-24.

6 Ch'en IY, Katz DS, Jeffrey RB Jr. Do arterial phase helical CT images improve detection or characterization of colorectal liver metastases? J Comput Assist Tomogr. 1997;21(3):391-7.

7 Choi J. Imaging of hepatic metastases. Cancer Control. 2006;13(1):6-12.

8 Choti MA, Bukley GB. Management of metastatic disease. In: Schiff ER, Sorrel MF, Maddrey WC, editors. Diseases of the liver. 8th ed. Philadelphia: LippincottRaven; 1999. p.1319-33.

9 Choti MA, Bulkley GB. Management of hepatic metastases. Liver Transplant Surg. 1999;5:65-80.

10 Delbeke D, Vitola JV, Sandler MP. Stanging recurrent metastatic colorectal carcinoma with PET. J Nucl Med. 1997;38:1196-201.

11 Eberhardt SC, Choi PH, Bach AM. Utility of sonography for small hepatic lesions found on computed tomography in patients with cancer. J Ultrasound Med. 2003;22:335-43; quiz 345-6.

12 Even-Sapir E, Parag Y, Lerman H. Detection of recurrence in patients with rectal cancer: $\mathrm{PET} / \mathrm{CT}$ after abdominoperineal or anterior resection. Radiology. 2004;232:815-22.

13 Fong Y, Cohen AM, Fortner JG. Liver resection for colorectal metastases. J Clin Oncol. 1997;15:938-46. 
14 Gambhir SS, Shepard JE, Shah BD. Analytical decision model for the cost-effective management of solitary pulmonary nodels. J Clin Oncol. 1998;16:2113-25.

15 Gayotto LCC. Metástases hepáticas. In: Gayotto LCC, Alves VAF, editores. Doenças do fígado e vias biliares. São Paulo: Atheneu; 2001. p.986-9.

16 Gold P, Freedman SO. Demonstration of tumorspecific antigens in human colonic carcinomata by immunological tolerance and absorption techniques. J Exp Med. 1965;121:439-62.

17 Gold P, Freedman SO. Specific carcinoembryonic antigens of the human digestive system. J Exp Med. 1965;122:467-81.

18 Hamm B, Mahfouz AE, Taupitz M. Liver metastases: improved detection with dynamic gadolinium-enhanced MR imaging? Radiology. 1997;202(3):677-82.

19 Hostetter RB, Augustus LB, Mankarious R, Chi K, Fan $D$, Toth C. Carcinoembryogenic antigen as a selective enhancer of colorectal cancer metastasis. J Natl Cancer Inst. 1990;82:380-5.

20 Hughes KS, Rosenstein RB, Songhorabodi S. Resection of the liver for colorectal carcinoma metastases. A multiinstitutional study of long-term survivors. Dis Colon Rectum. 1988;31:1-4.

21 Instituto Nacional do Câncer (INCA). Estimativas da incidência e mortalidade por câncer no Brasil em 2006 [citado em 6 mar. 2008]. Disponível em: http://www.inca. gov.br.

22 Juweid ME, Cheson S, Johnson G, Schuchter LM, Zhuang $\mathrm{H}$, Alavi A. Is 18F-FDG PET more accurate than standard diagnostic procedures in the detection of suspected recurrent melanoma? J Nucl Med. 2004;45:1323-7.

23 Mineo TC, Ambrogi V, Tonini G. Longterm results after resection of simultaneous and sequential lung and liver metastases from colorectal carcinoma. J Am Coll Surg. 2003;197:386-91.

24 NHS Executive. Guindace on commissioning cancer services: improving outcomes in colorectal cancer: the research evidence. London: Departament of Health; 1997.

25 Nordlinger B, Guiguet M, Vaillant JC. Surgical resection of colorectal carcinoma metastases to the liver. A prognostic scoring system to improve case selection, based on 1568 patients. Association Francaise de Chirurgie. Cancer. 1996;77:1254-62.

26 Office of National Statistics. Cancer survival in health authorities of England, 1993-2000. Health Stat Quart. 2002;13:95-103.

27 Reske SN, Kotzerke. Result of the $3^{\text {rd }}$ Gereman Interdiciplinary Consensus Conference, "Onco-PET 111. Eur J Nucl Med. 2001;28:1707-23.

28 Rigo P, Paulus P, Kaschten BJ. Oncological applications of positron emission tomography with fluorine-18 fluorodeoxyglucose. Eur J Nucl Med. 1996;23:1641-4.

29 Robinson PJ. Indeterminate liver lesions in cancer. Cancer Imaging. 2002;2:130-2.

30 Rydzewski B, Dehdashti F, Gordon BA. Usefulness of intraoperative sonography for revealing hepatic metastases from colorectal cancer in patients selected for surgery after undergoing FDG/PET. AJR Am J Roentgenol. 2002;178(2):353-8.

31. Scheele J, Stang R, Altendorf-Hofmann A, Paul M. Resection of colorectal liver metastases. World J Surg. 1995;19:59-71.

32. Schima W, Kulinna C, Langerberger H, Ba-Ssalamah A. Liver metastases of colorectal cancer: US, CT or MR? Cancer Imaging. 2005;5:S149-S155.

33. Sherlock S, Dooley J. Tumores malignos do fígado. In: Sherlock S, Dooley J, editores. Doenças do fígado e do sistema biliar. 11a ed. Rio de Janeiro: Guanabara; 2004. p.461-79.

34. Soyer P, de Givry SC, Gueye C. Detection of focal hepatic lesions with MR imaging: prospective comparison of T2-weighted fast spin-echo with and without fat suppression, T2-weighted breath-hold fast spin-echo, and gadolinium-enhanced 3D gradient-recalled imaging. AJR Am J Roentgenol. 1996;166(5):1115-21.

35. The management of colorectal cancers. Effective Health Care. 2004;8(6) [Cited 2007 March 26]. Available from: http://www.york.ac.uk/inst/crd/ehc83.pdf.

36. Vauthey JN, Choti MA, Helton WS. AHPBA/SSO/ SSAT Consensus Conference on Hepatic Colorectal Metastases: Rationale and Overview of the Conference. Ann Surg Oncol. 2006;13(10):1259-60.

37. Vitola JV, Delbeke D, Sandler MP. Positron emission tomography to stage suspected metastatic colorectal carcinoma to the liver. Am J Surg. 1996;171:21-6.

38. von Herbay A, Vogt C, Willers R, Haussinger. Real-time imaging with the sonographic contrast agent SonoVue: differention between benign and malignant hepatic lesions. J Ultrasound Med. 2004;23:1557-68.

39. Ward J, Naik KS, Guthrie JA. Hepatic lesion detection: comparison of MR imaging after superparamagnetic iron oxide with dual-phase CT by using alternative free response receiver operating characteristics analysis. Radiology. 1999;210(2):459-66.

40. Watson AJM, Lolohea S, Robertson GM, Frizelle FA. The role of positron emission tomography in the management of recurrent colorectal cancer: a review. Dis Colon Rectum. 2006;50:102-14.

41. Zerhouni EA, Rutter C, Hamilton SR. CT and MR imaging in the staging of colorectal carcinoma: report of the radiology diagnostic oncology group II. Radiology. 1996;200(2):443-51. 